\title{
MEANING-REFINING ACOUSTIC VARIATION WITHIN THE INTERNAL STRUCTURE OF PIED BABBLER RECRUITMENT CRIES
}

\author{
SABRINA ENGESSER ${ }^{* 1}$, AMANDA R. RIDLEY ${ }^{2}$, MARTA B. MANSER ${ }^{3}$, and \\ SIMON W. TOWNSEND ${ }^{1,4}$ \\ *Corresponding Author: sabrina.engesser@uzh.ch \\ ${ }^{1}$ Department of Comparative Linguistics, University of Zurich, Zurich, Switzerland \\ ${ }^{2}$ Centre for Evolutionary Biology, The University of Western Australia, \\ Crawley, Australia \\ ${ }^{3}$ Department of Evolutionary Biology and Environmental Studies, University \\ of Zurich, Switzerland \\ ${ }^{4}$ Department of Psychology, University of Warwick, Coventry, UK
}

Human language is a generative system that achieves its limitless productivity by assembling sounds into larger meaningful constructs (Chomsky, 1981; Hockett, 1960). Comparative work has demonstrated animals can also combine sounds in ways analogous to humans and this has shed important light on the prevalence of combinatorial capacities outside of humans (primates: Arnold \& Zuberbühler, 2006; Ouattara, Lemasson, \& Zuberbühler, 2009 / birds: Engesser et al., 2015; Engesser et al., 2016; Pepperberg, 2007; Suzuki et al., 2016 / see also: Hurford, 2007; Marler, 1977). However, further analogue examples are central in unveiling the distribution and diversity of combinatorial mechanisms in non-human communication systems, and to identify potential factors driving their emergence (Schlenker, Chemla, \& Zuberbühler, 2016).

Here we present evidence for a novel form of combinatorial structuring in the vocal system of a highly social passerine with a fixed vocal repertoire, the southern pied babbler (Turdoides bicolor) (Ridley \& Raihani, 2007). By studying a population of wild, but habituated, babblers we demonstrate that male babblers produce two variants of long and raucous, 'cry-like' structures, which generally appear to function to recruit group members during group travel. Using acoustic analyses, we show that both cry variants are similar in their 
super-structure, but differ in their sub-structure. Preceded by a wind-up segment, they either grade into repetitions of $\mathrm{A} /$ single-note or $\mathrm{AB} /$ double-note motifs, with the same A-notes being shared across the two variants (Fig. 1). Behavioural observations on the natural occurrence of the two cry types in combination with playback experiments indicated that, consistent with similarities in their superstructure, both types function overall in recruiting group members during locomotion, but the internal $\mathrm{A}$ or $\mathrm{AB}$ sub-structure specifies the precise form of recruitment. Specifically, in response to A/single-note cries receivers were found to approach to the caller's announced location (i.e. tantamount to 'come to me'), while $\mathrm{AB}$ /double-note cries were associated with caller movement and with receivers following the caller over long distances (i.e. tantamount to 'come with me'). We suggest that the overall structure of the two cry variants likely conveys the same intention of the caller to recruit its group members, with the internal motif pattern refining the signal's functional specificity. Accordingly, the $\mathrm{B}$ note might represent an acoustic modifier altering or intensifying the A note's meaning.

We argue the pied babbler recruitment cry represents another intriguing example illustrating the variability of generative mechanisms outside of human language. Our work lends support to the hypothesis that combinatoriality emerged in species with constrained sound repertoires, whereby the assemblage of sounds into more distinctive structures might enhance signal discrimination, and hence increase communicative output (Arnold \& Zuberbühler, 2008; Nowak \& Krakauer, 1999). Ultimately, by unveiling potential conditions promoting the emergence of combinatorial capacities, such comparative data on non-human animals can provide valuable insights into the evolutionary progression of our own language system.

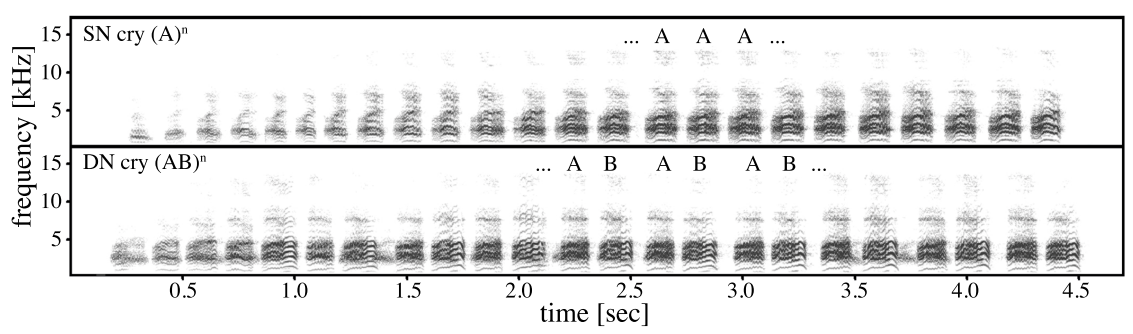

Figure 1. Spectrograms of a single-note (SN) and a double-note (DN) recruitment cry of one dominant male babbler. Capital letters denote the note type. 


\section{References}

Arnold, K., \& Zuberbühler, K. (2006). Language evolution: Semantic combinations in primate calls. Nature, 441(7091), 303.

Arnold, K., \& Zuberbühler, K. (2008). Meaningful call combinations in a nonhuman primate. Current Biology, 18(5), R202-R203.

Chomsky, N. (1981). Knowledge of Language: Its Elements and Origins. Philosophical Transactions of The Royal Society B: Biological Sciences, 295(1077), 223-234.

Engesser, S., Crane, J. M., Savage, J. L., Russell, A. F., \& Townsend, S. W. (2015). Experimental Evidence for Phonemic Contrasts in a Nonhuman Vocal System. PloS Biology, 13(6), e1002171.

Engesser, S., Ridley, A. R., \& Townsend, S. W. (2016). Meaningful call combinations and compositional processing in the southern pied babbler. Proceedings of the National Academy of Sciences, 113(21), 5976-5981.

Hockett, C. F. (1960). The Origin of Speech. Scientific American, 203, 88-111.

Hurford, J. (2007). The origins of meaning. Oxford: Oxford University Press.

Marler, P. (1977). The structure of animal communication sounds. Recognition of complex acoustic signals: report of Dahlem workshop. Berlin: Abakon Verlagsgesellschaft.

Nowak, M. A., \& Krakauer, D. C. (1999). The evolution of language. Proceedings of the National Academy of Sciences, 96(14), 8028-8033.

Ouattara, K., Lemasson, A., \& Zuberbühler, K. (2009). Campbell's monkeys concatenate vocalizations into context-specific call sequences. Proceedings of the National Academy of Sciences, 106(51), 22026-22031.

Pepperberg, I. M. (2007). Grey parrots do not always 'parrot': the roles of imitation and phonological awareness in the creation of new labels from existing vocalizations. Language Sciences, 29(1), 1-13.

Ridley, A. R., \& Raihani, N. J. (2007). Variable postfledging care in a cooperative bird: causes and consequences. Behavioral Ecology, 18(6), 9941000 .

Schlenker, P., Chemla, E., \& Zuberbühler, K. (2016). What Do Monkey Calls Mean? Trends in Cognitive Sciences, 20(12), 894-904.

Suzuki, T. N., Wheatcroft, D., \& Griesser, M. (2016). Experimental evidence for compositional syntax in bird calls. Nature Communications, 7, 10986. 